\title{
Selective activation of inflammation factors by human parvovirus B19 and human bocavirus VP1 unique region on H9c2 cardiomyocyte
}

\author{
HIONG-PING HII ${ }^{1}$, CHUN-CHING CHIU ${ }^{2,3}$, DI-WEI LIN ${ }^{3}$, YA-FANG SHI ${ }^{3}$, \\ TSAI-CHING HSU ${ }^{3-5}$ and BOR-SHOW TZANG ${ }^{3-6}$
}

\author{
${ }^{1}$ Division of Cardiovascular, Department of Surgery, Chi Mei Medical Center, Tainan 710; \\ ${ }^{2}$ Department of Neurology and Medical Intensive Care Unit, Chunghua Christian Hospital, Chunghua 505; \\ ${ }^{3}$ Institute of Biochemistry, Microbiology and Immunology; ${ }^{4}$ Clinical Laboratory, \\ Chung Shan Medical University Hospital; ${ }^{5}$ Immunology Research Center; ${ }^{6}$ Department of Biochemistry, \\ School of Medicine, Chung Shan Medical University, Taichung 402, Taiwan, R.O.C.
}

Received April 3, 2018; Accepted August 2, 2018

DOI: $10.3892 / \mathrm{mmr} .2018 .9369$

\begin{abstract}
Human parvovirus B19 (B19) and human bocavirus $1(\mathrm{HBoV})$ are the only known pathogenic parvoviruses, and are responsible for a variety of diseases in human beings. Mounting evidence indicates a strong association between B19 infection and cardiac disorders including myocarditis, dilated cardiomyopathy and heart failure. However, very limited information about the role of $\mathrm{HBoV}$ in cardiac disorders is known. To elucidate the effects of $\mathrm{B} 19$ and $\mathrm{HBoV}$ on cardiac disorders, we expressed EGFP-conjugate constructs of B19-VP1 unique region (VP1u) and $\mathrm{HBoV}-\mathrm{VP} 1 \mathrm{u}$, along with the mutants EGFP-B19-VP1uD175A and EGFP-HBoV-VP1uV12A, in $\mathrm{H} 9 \mathrm{c} 2$ cells by stable transfection. The protein expression levels of EGFP, EGFP-B19-VP1u, EGFP-B19-VP1uD175A, EGFP-HBoV-VP1u and EGFP-HBoV-VP1uV12A in H9c2 cells were observed under a fluorescence microscope and confirmed by western blotting. Secreted phospholipase A2 (sPLA2) activity was detected in B19-VP1u and HBoV-VP1u but not B19-VP1uD175A and HBoV-VP1uV12A recombinant proteins. Significantly higher expression levels of MCP2 and IP-10 mRNA were detected in H9c2 cells that were transfected with pEGFP-B19-VP1u, compared with in those cells transfected with pEGFP-HBoV-VP1u, pEGFP-B19-VP1uD175A or
\end{abstract}

Correspondence to: Dr Bor-Show Tzang, Department of Biochemistry, School of Medicine, Chung Shan Medical University, No. 110, Section 1, Jianguo North Road, Taichung 40201, Taiwan, R.O.C.

E-mail: bstzang@csmu.edu.tw

Dr Tsai-Ching Hsu, Institute of Biochemistry, Microbiology and Immunology, Chung Shan Medical University, No. 110, Section 1, Jianguo North Road, Taichung 40201, Taiwan, R.O.C.

E-mail: htc@csmu.edu.tw

Key words: human parvovirus B19, human bocavirus 1, VP1 unique region, cardiac inflammation
pEGFP-HBoV-VP1uV12A. Significantly higher protein levels of IL-1 $\beta$ and IL-6 were detected in H9c2 cells transfected with pEGFP-B19-VP1u or pEGFP-HBoV-VP1u, compared with in those cells transfected with pEGFP-B19-VP1uD175A or pEGFP-HBoV-VP1uV12A. Notably, significantly higher expression of both TNF- $\alpha$ and NF- $\kappa \mathrm{B}$ was observed only in $\mathrm{H} 9 \mathrm{c} 2$ cells transfected with pEGFP-B19-VP1u, but not in those cells transfected with pEGFP-HBoV-VP1u, pEGFP-B19-VP1uD175A or pEGFP-HBoV-VP1uV12A. These findings, to our knowledge for the first time, reveal the difference between B19-VP1u and HBoV-VP1u in H9c2 cells and provide insight into the roles of B19-VP1u and HBoV-VP1u in the pathogenesis of cardiac inflammation.

\section{Introduction}

Human parvovirus B19 (B19) and human bocavirus 1 (HBoV) are the only known pathogenic parvoviruses in human beings $(1,2)$. B19, discovered in 1975 by Cossart during screening for hepatitis B virus (3), is highly infectious and causes various pathological conditions including fifth disease in children, persistent anemia in immunocompromised patients, transient aplastic crises, hydrops fetalis in pregnant women, arthropathy, and autoimmune diseases (4-7). HBoV, discovered in 2005 by Allender et al (8), was first identified in the respiratory nasopharyngeal aspirates of children with lower respiratory tract infections. $\mathrm{HBoV}$ is known to be a significant causative agent in acute respiratory tract infections, in which wheezing is the most common symptom (9).

In the literature, intra-nuclear viral particles typical of B19 were first documented in an electron microscopic examination of fetal cardiac tissue (10); however, B19 is not regarded as a cardiotropic virus (11). Notably, a previous investigation identified B19 as a pathogenic agent in cases of myocarditis in children and adolescents (12). More recently, an increasing body of evidence has shown that B19 is strongly associated with cardiovascular disorders. In the last decade, B19 has emerged as a potential pathogenic agent in adult patients with 
inflammatory heart disease (13). Indeed, the B19 genome has been detected in endomyocardial biopsies of patients with acute myocardial infarction (14). Another investigation found that the persistence of B19 may be associated with progression of left ventricular dysfunction (15). In a study of 208 patients, dominantly higher prevalence of the B19 genome was found in endomyocardial biopsies of inpatients with inflammatory cardiomyopathy or myocarditis compared with controls (16). These findings strongly indicate a connection between B19 and heart disorders.

Phospholipase A2 (PLA2)-like activity of B19-VP1 unique region (VP1u) has been identified (17) and associated with its infectivity and the pathogenesis of many disorders (18-21). Recently, B19-VP1u has been associated with cardiac disorders $(22,23)$. Abnormal ultrastructural changes in the myocardia and elevated levels of myocardial functional enzymes, including aspartate aminotransferase (AST), lactate dehydrogenase (LDH), creatine kinase (CK), creatine kinase isoenzyme (CK-MB) and alpha-hydroxybutyric acid dehydrogenase (alpha-HBDH), have been detected in mice receiving recombinant B19-VP1u proteins (22). Similarly, dilated cardiomyopathy has been observed in BALB/c mice immunized with VPlu; an observation clinically relevant to B19-associated cardiac damage (24). Although a recent investigation indicated that HBoV-VP1u also has a PLA2 motif, and could exhibit sPLA2 activity (25), little is known about the role of HBoV-VP1u in cardiac injury. Therefore, this study compared B19-VP1u and HBoV-VP1u with respect to their potential roles in inducing injury in $\mathrm{H} 9 \mathrm{c} 2$ cardiomyocytes.

\section{Materials and methods}

Plasmids. Plasmid pEGFP-C1 was purchased from CLONTECH (Clontech Laboratories, Inc., Mountainview, CA, USA). The B19-VP1u and B19-VP1uD175A genes described in our previous study (26) were ligated into the pEGFP-C1 expression vector, which are known as pET32a-B19-VP1u and pET32a-B19-VP1uD175A. A 387-bp DNA fragment encompassing nucleotides 3056-3442 of the Taiwan HBoV strain (TW125_07: GeneBank accession no. EU984241.1) provided by Centers for Disease Control, Taipei, Taiwan (27) was amplified by the polymerase chain reaction (PCR) using the following primers, including 5'-GCA GATCTATGCCTCCAATTAAG-3' (forward primer) and 5'-GCGTCGACTGAGGTTCCTGG-3' (reverse primer). The HBoV-VPlu and HBoV-VP1uV12A, the mutant form of HBo-VP1u without sPLA2 activity $(25,28)$, were constructed into pEGFP-C1, which are known as pET32a-HBoV-VP1u and pET32a-HBoV-VP1uV12A. All constructants were verified by DNA sequencing analysis forwardly and reversely.

Secreted SPLA2 catalytic activity. The secreted SPLA2 activity was detected as cribbed elsewhere (27). The recombinant protein samples, including B19-VP1u, B19-VP1uD175A, HBoV-VP1u and HBoV-VP1uV12A, were assayed for sPLA2 activity by using a colorimetric assay (cat. no. 765001, sPLA2 Activity kit; Cayman Chemical), in accordance with the manufacturer's instructions, with dynamic colorimetric measurements at the optical density of $414 \mathrm{~nm}$ determined every minute for $10 \mathrm{~min}$. Results are revealed as micromoles per minute per milliliter.

Cell culture, transfection and stable clones. H9c2 cardiac myoblast cells were purchased from ATCC and cultured in Dulbecco's modified Eagle's medium (DMEM) supplemented with $10 \%$ fetal bovine serum (FBS; Gibco; Thermo Fisher Scientific, Inc., Waltham, MA, USA) at $37^{\circ} \mathrm{C}$ in a $5 \%$ $\mathrm{CO}_{2}$ incubator. A total of $1 \times 10^{6}$ cells were grown to $70-80 \%$ confluence in a $100 \mathrm{~mm}^{2}$ dish before transfection. The transfection reaction was performed using Lipofectamine reagent with PLUS reagent (Invitrogen; Thermo Fisher Scientific, Inc.) with $2 \mu \mathrm{g}$ of the plasmids, pEGFP, pEGFP-B19-VP1u, pEGFP-B19-VP1uD175A (a mutant of the B19-VP1u region), pEGFP-HBoV-VP1u and pEGFP-HBoV-VP1uV12A (a mutant of HBoV-VP1u region), respectively. To determine the transfection efficiency, pCMV-SPORT- $\beta$-gal plasmid (0.5 $\mu \mathrm{g} /$ transfection, Invitrogen; Thermo Fisher Scientific, Inc.) and co-transfected with the constructs mentioned above, respectively. After X-gal staining (29), cells were fixed with chilled methanol and the extent of $\beta$-gal expression was measured by determining the ratio of the X-gal-stained area to the area of each observation field under a light microscope. No significant variation was observed among all groups (data not shown). The cells were then cultured in serum-free DMEM for $12 \mathrm{~h}$ at $37^{\circ} \mathrm{C}$ in a $5 \% \mathrm{CO}_{2}$ incubator and subsequently in DMEM with $10 \%$ FBS. The stable clones were obtained by G418 selection at a concentration of $600 \mathrm{mg} / \mathrm{ml}$ (Promega Corporation, Madison, WI, USA) in DMEM containing $10 \%$ FBS for 8 weeks. The expression levels of EGFP and the EGFP-B19-VP1u,EGFP-B19-VP1uD175A, EGFP-HBoV-VP1u and EGFP-HBoV-VPluV12A fusion proteins were examined by using a Zeiss Axioplan-2 epifluorescence microscope (Carl Zeiss AG, Oberkochen, Germany) and by western blot analysis.

Fluorescence microscopy. Expression of recombinant EGFP, EGFP-B19-VP1u, EGFP-B19-VP1uD175A, EGFP-HBoV-VP1u and EGFP-HBoV-VP1uV12A in H9c2 cells was observed with a Zeiss Axioplan-2 fluorescence microscope (Carl Zeiss AG). Transfected H9c2 cells were fixed by $4 \%$ paraformaldehyde, permeabilized with $0.5 \%$ Triton X-100, and blocked with $1 \%$ BSA in phosphate-buffered saline (PBS) for $10 \mathrm{~min}$. The cell nuclei were stained with DAPI (blue). Excitation filters/emission filters were set at 480/535 and 358/460 nm for green fluorescent protein (GFP) and DAPI, respectively. Digital images of the cells were recorded by using a spot camera system.

Reverse transcription-semi quantitative (RT-sq)PCR. All procedures were carried out in a designated PCR clean area. RNA was extracted from infected cells using Trizol reagent (Invitrogen; Thermo Fisher Scientific, Inc.). Total RNA was isolated from the cells transfected with EGFP, EGFP-B19-VP1u, EGFP-B19-VP1uD175A, EGFP-HBoV-VP1u and EGFP-HBoV-VP1uV12A, respectively. The RNA samples were suspended in diethyl pyrocarbonate (DEPC)-treated water, quantified, and then stored at $-80^{\circ} \mathrm{C}$ until use. RNA concentration and purity were determined by a spectrophotometer by calculating the ratio of optical density at wavelengths of 260 and $280 \mathrm{~nm}$. The first-strand cDNA for PCR was synthe- 
A
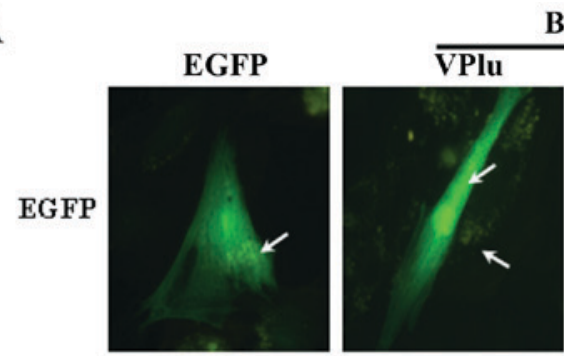

B19V
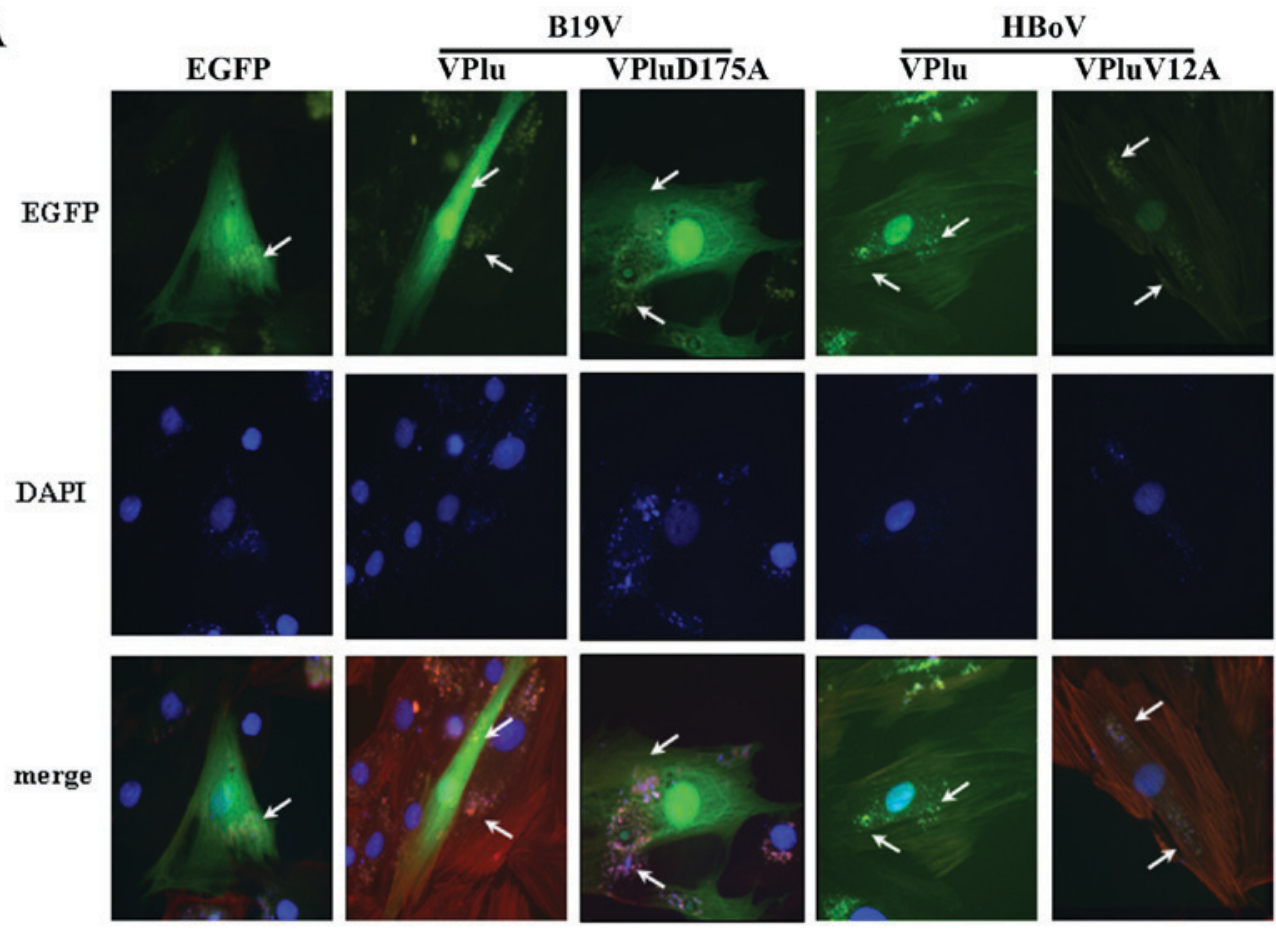

B

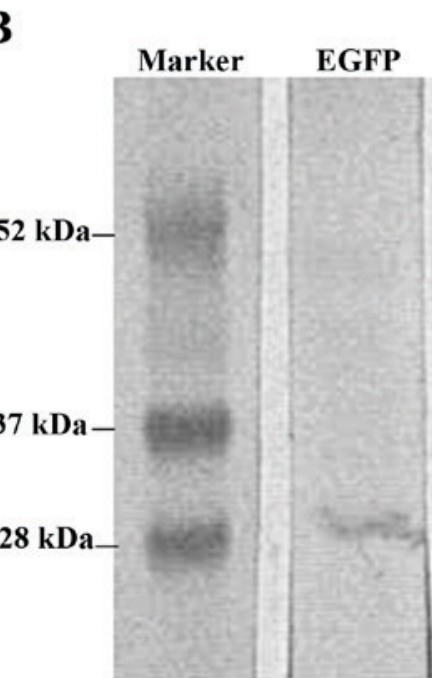

B19V

HBoV

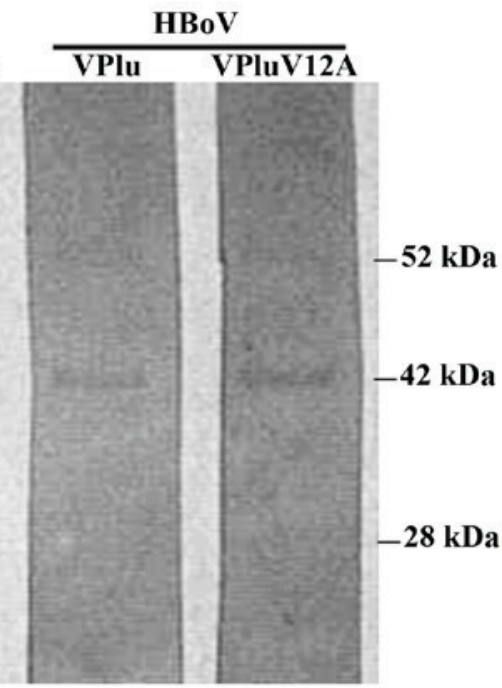

Figure 1. Expression of EGFP, EGFP-B19-VP1u, EGFP-B19-VP1uD175A, EGFP-HBoV-VP1u and EGFP-HBoV-VP1uV12A in H9c2 cells. (A) Representative images of H9c2 cells transfected with pEGFP, pEGFP-B19-VP1u, pEGFP-B19-VP1uD175A, pEGFP-HBoV-VP1u or pEGFP-HBoV-VP1uV12A, observed under a fluorescence microscope. EGFP expression is indicated with white arrows (magnification, x200). (B) Expression of EGFP, EGFP-B19-VP1u, EGFP-B19-VP1uD175A, EGFP-HBoV-VP1u and EGFP-HBoV-VP1uV12A recombinant proteins was detected using antibodies against EGFP. Similar results were observed in triplicate experiments.

sized from total RNA $(2 \mu \mathrm{g})$ using the ImProm-II Reverse Transcription System (Promega Corporation). The cDNAs encoding monocyte chemoattractant protein 2 [MCP2, also known as chemokine ligand 8 (CCL-8)], IFN-gamma-inducible protein 10 [IP-10, also known as CSC motif chemokine 10 (CXCL10)] and GAPDH were amplified by using a multiplex PCR kit (cat. no. MP-70070; Maxim Biotech, Inc., Rockville, MD, USA). The intensity of MPC2, IP-10 and GAPDH were then quantified using densitometric apparatus (Alpha-Imager 2200; ProteinSimple, San Jose, CA, USA).

Protein extraction. The cells were centrifuged at $800 \mathrm{~g}$ for 5 min and washed twice with ice-cold PBS twice. The cell pellets were then suspended in $600 \mu$ l of PRO-PREP ${ }^{\mathrm{TM}}$ buffer
(iNtRON Biotech, Gyeonggi-do, Korea) and chilled on ice for $1 \mathrm{~h}$. The supernatant containing protein extracts were then collected by centrifugation at $17,982 \mathrm{~g}$ for $5 \mathrm{~min}$ at $4^{\circ} \mathrm{C}$. Protein concentration of the samples was determined by a modified Bradford assay using a spectrophotometer (Hitachi U3000; Hitachi, Ltd., Tokyo, Japan) at $595 \mathrm{~nm}$ with BSA as the standard.

Immunoblotting. Protein samples were separated by 10 or $12 \%$ SDS-PAGE and electrophoretically transferred to nitrocellulose membranes (GE Healthcare, Chicago, IL, USA). After blocking with 5\% non-fat dry milk in PBS, antibodies against GFP (cat. no. 460092; Invitrogen; Thermo Fisher Scientific, Inc.), TNF- $\alpha$ (cat. no. sc-8301), IL-6 (cat. no. sc-1265), IL-1 $\beta$ 
(cat. no. sc-7884), NF-кB p65 (cat. no. sc-109) (1:500-1:1,000; Santa Cruz Biotechnology, Inc., Dallas, TX, USA) and $\beta$-actin (1:5,000; MAB1501, Chemicon; EMD Millipore, Billerica, MA, USA) were diluted in PBS with $2.5 \%$ BSA and incubated with the membranes for $1.5 \mathrm{~h}$ with gentle agitation at room temperature. The membranes were then incubated with horseradish peroxidase (HRP)-conjugated secondary antibody (cat. nos. sc-2004 or sc-2005; Santa Cruz Biotechnology, Inc.). Immobilon Western Chemiluminescent HRP Substrate (EMD Millipore) and a chemiluminescence imaging analyzer (GE ImageQuant TL 8.1; GE Healthcare Bio-Sciences, Pittsburgh, PA, USA) were used to detect the antigen-antibody complexes. The blotting results were then quantified using densitometric apparatus (Alpha-Imager 2200; ProteinSimple).

Statisticalanalysis.Allof the statisticalanalyseswere performed using GraphPad Prism 5 software (GraphPad Software, Inc., La Jolla, CA, USA) by one-way analysis of variance (One-way ANOVA) followed by Tukey's multiple-comparisons test. Data were represented as mean \pm SEM and verified at least three independent experiments. $\mathrm{P}<0.05$ was considered to indicate a statistically significant difference. The significant differences were stressed with symbols as shown in figures.

\section{Results}

Expression of recombinant B19-VPlu and HBoV-VPlu. To detect the expression of the recombinant EGFP, EGFP-B19-VP1u,EGFP-B19-VP1uD175A,EGFP-HBoV-VP1u and EGFP-HBoV-VP1uV12A proteins in H9c2 cells, an epifluorescence microscope and western blot analysis were employed. The upper panel of Fig. 1A shows photographs of H9c2 cells that were transfected with pEGFP, pEGFP-B19-VP1u, pEGFP-B19-VP1uD175A, pEGFP-HBoV-VP1u and pEGFP-HBoV-VP1uV12A. The expressions of EGFP, EGFP-B19-VP1u,EGFP-B19-VP1uD175A,EGFP-HBoV-VP1u and EGFP-HBoV-VP1uV12A was observed as indicated by the arrow in the figure. The nuclei of $\mathrm{H} 9 \mathrm{c} 2$ cells were stained with DAPI. The expression of the EGFP, EGFP-B19-VP1u, EGFP-B19-VP1uD175A, EGFP-HBoV-VP1u and EGFP-HBoV-VP1uV12A recombinant proteins was further verified using antibodies against EGFP (Fig. 1B).

Secreted sPLA2 activity of recombinant B19-VP1u and $H B o V-V P 1 u$. To determine the sPLA2 catalytic activity of the purified recombinant proteins, an sPLA2 activity assay was performed. Table I summarizes the sPLA2 activities in B19-VP1u, B19-VP1uD175A, HBoV-VP1u and $\mathrm{HBoV}-\mathrm{VP} 1 \mathrm{uV} 12 \mathrm{~A}$ recombinant proteins. As a positive control, bvPLA2 exhibited an sPLA2 activity of $0.368 \pm 0.009 \mu \mathrm{mol} / \mathrm{min} / \mathrm{ml}$, whereas B19-VP1uD175A and HBoV-VP1uV12A exhibited no detectable sPLA2 activities. Accordingly, sPLA2 activities were detected for B19-VP1u (400 ng) and HBoV-VPlu (400 ng) at the values of $0.098 \pm 0.012$ and $0.046 \pm 0.007 \mu \mathrm{mol} / \mathrm{min} / \mathrm{ml}$, respectively (Table I).

Effects of recombinant B19-VP1u and HBoV-VP1u on chemokine expression in $H 9 c 2$ cells. To study the effects of EGFP, EGFP-B19-VP1u, EGFP-B19-VP1uD175A, EGFP-HBoV-VP1u and EGFP-HBoV-VP1uV12A on chemo-
Table I. Determination of sPLA2 activity.

Proteins

sPLA2 activity ( $\mathrm{mmol} / \mathrm{min} / \mathrm{ml})$

bvPLA2 (10 ng)
B19-VP1u (400 ng)
B19-VP1uD175A (400 ng)
HBoV-VP1u (400 ng)
HBoV-VP1uV12A (400 ng)

$0.368 \pm 0.009$

$0.098 \pm 0.012$

ND

$0.046 \pm 0.007$

ND

Similar results were observed in triplicate experiments $(\mathrm{N}=3)$ and the data was expressed with mean \pm SEM. bvPLA2, sPLA2 from bee venom PLA2 control; ND, no detected; sPLA2, secreted phospholipase A2.
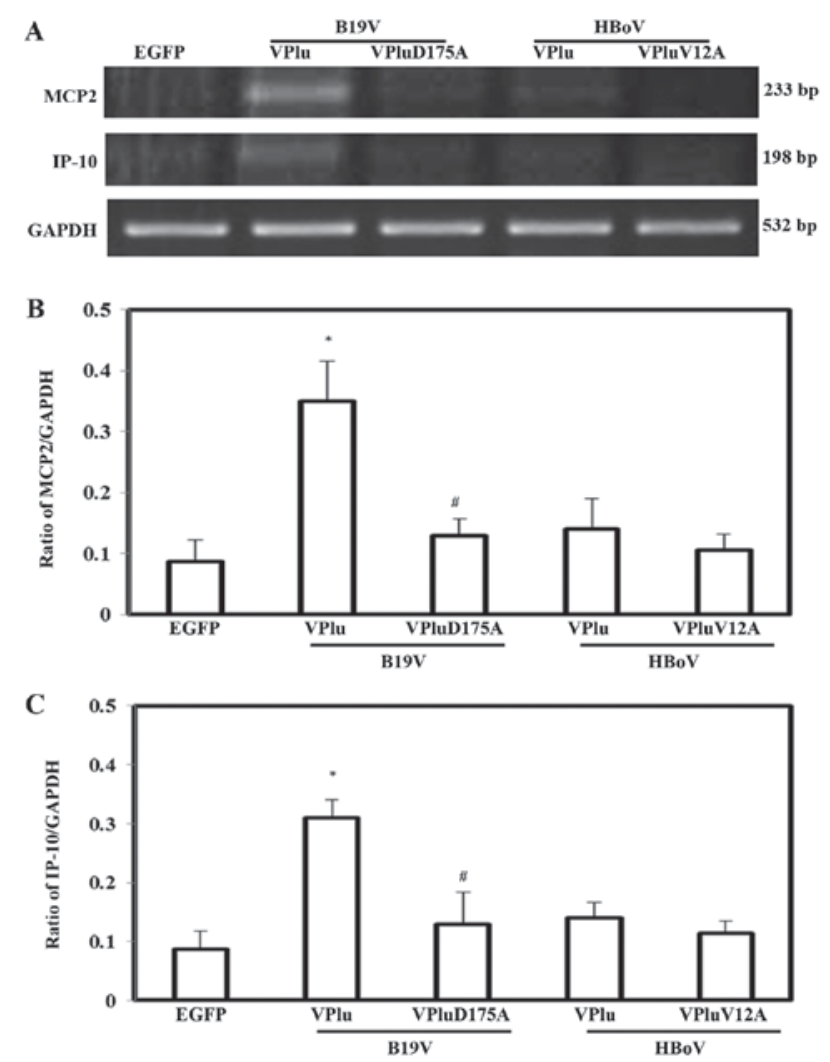

Figure 2. Expression of MCP2 and IP-10 mRNA. (A) Total mRNA of MCP2 and IP-10 in H9c2 cells transfected with pEGFP, pEGFP-B19-VP1u, pEGFP-B19-VP1uD175A, pEGFP-HBoV-VP1u or pEGFP-HBoV-VP1uV12A, detected by reverse transcription-semi quantitative polymerase chain reaction. Relative levels of (B) MCP2 and (C) IP-10 are shown based on GAPDH level. Similar results were observed in triplicate experiments. ${ }^{*} \mathrm{P}<0.05$, relative to the EGFP transfection group and ${ }^{\#} \mathrm{P}<0.05$, relative to the $\mathrm{B} 19 \mathrm{~V}-\mathrm{VP} 1 \mathrm{u}$ transfection group.

kine expression, the mRNA expressions of MCP2 and IP-10 were measured (Fig. 2). Significantly higher levels of MCP2 and IP-10 mRNA were detected in H9c2 cells transfected with pEGFP-B19-VP1u, but not in those cells transfected with pEGFP-HBoV-VP1u, pEGFP-B19-VP1uD175A or pEGFP-HBoV-VP1uV12A, relative to expression in cells transfected with pEGFP. Quantified results of MCP2 and IP-10 mRNA levels based on GAPDH level are shown in Fig. 2B and C. 

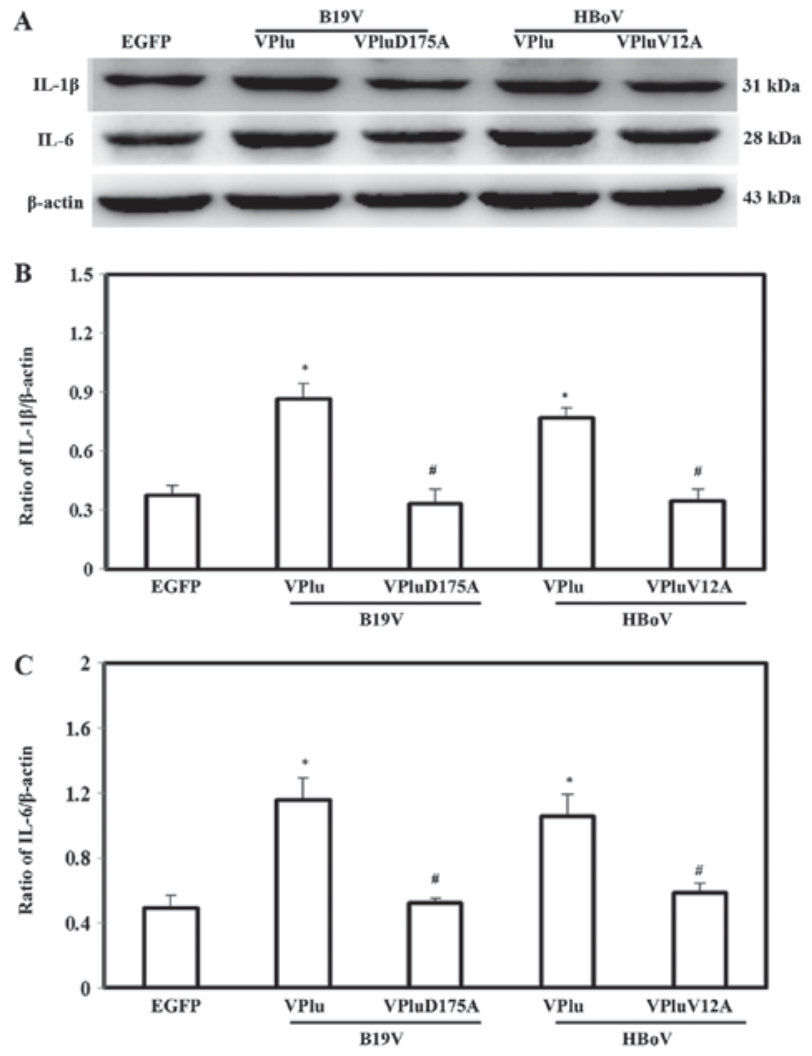

Figure 3. Expression of IL-1 $\beta$ and IL-6 proteins. (A) IL-1 $\beta$ and IL-6 expression in H9c2 cells transfected with pEGFP, pEGFP-B19-VP1u, pEGFP-B19-VP1uD175A, pEGFP-HBoV-VP1u or pEGFP-HBoV-VP1uV12A, detected by immunoblotting. Relative levels of (B) IL-1 $\beta$ and (C) IL-6 are presented based on $\beta$-actin expression. Similar results were observed in triplicate experiments. "P $<0.05$, relative to the EGFP transfection group and ${ }^{\text {"P }} \mathrm{P}<0.05$, relative to the B19V-VPlu transfection group.

Effects of recombinant B19-VPIu and HBoV-VPIu on inflammatory cytokines expression in $H 9 c 2$ cells. To examine the effects of EGFP-B19-VP1u, EGFP-B19-VP1uD175A, EGFP-HBoV-VP1u and EGFP-HBoV-VP1uV12A on the expressions of proinflammatory cytokines, IL-1 $\beta$, IL-6 and TNF- $\alpha$ levels were detected by western blotting. Significantly higher protein expression of IL- $1 \beta$ and IL- 6 was detected in H9c2 cells transfected with pEGFP-B19-VP1u and pEGFP-HBoV-VP1u, respectively, compared with in those cells transfected with pEGFP. Conversely, no significant difference in the expression of either IL- $1 \beta$ or IL- 6 was detected in H9c2 cells transfected with pEGFP-B19-VP1uD175A and pEGFP-HBoV-VP1uV12A, respectively, from that in cells transfected with pEGFP. The lower panel in Fig. 3 shows quantitative results concerning the expression levels of IL-1 $\beta$ and IL- 6 normalized to $\beta$-actin. Notably, significantly higher expression of TNF- $\alpha$ was observed only in H9c2 cells transfected with pEGFP-B19-VP1u, and not in those cells transfected with pEGFP-HBoV-VP1u, pEGFP-B19-VP1uD175A or pEGFP-HBoV-VP1uV12A, relative to that in cells transfected with pEGFP. The lower panel in Fig. 4 shows quantitative results concerning TNF- $\alpha$ levels normalized to $\beta$-actin. To identify the potential signaling involved in B19-VPlu-mediated cytokine induction, the expression of NF- $\kappa B$ was detected. Significantly higher expression of $\mathrm{NF}-\kappa \mathrm{B}$ protein was detected in $\mathrm{H} 9 \mathrm{c} 2$ cells transfected with pEGFP-B19-VP1u, compared with in
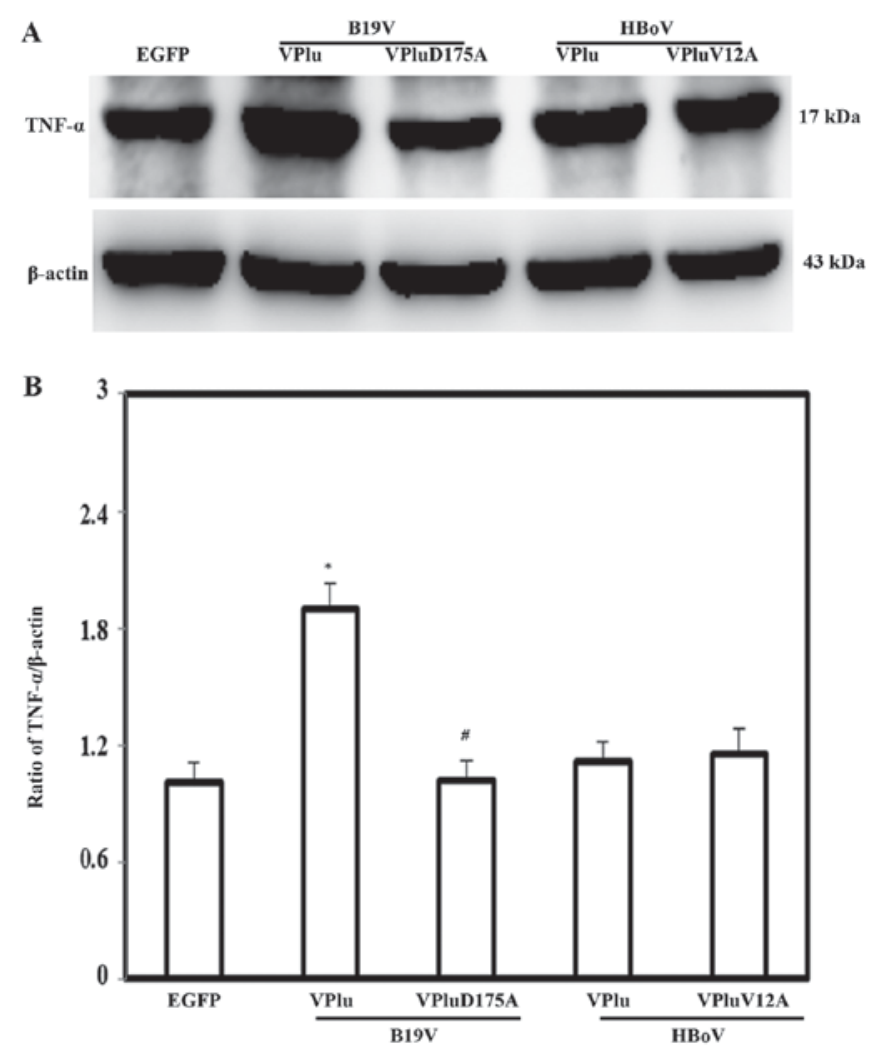

Figure 4. Expression of TNF- $\alpha$ protein. (A) TNF- $\alpha$ expression in H9c2 cells transfected with pEGFP, pEGFP-B19-VP1u, pEGFP-B19-VP1uD175A, pEGFP-HBoV-VP1u or pEGFP-HBoV-VP1uV12A, detected by immunoblotting. (B) The relative level of TNF- $\alpha$ is presented based on $\beta$-actin expression. Similar results were observed in triplicate experiments. ${ }^{*} \mathrm{P}<0.05$, relative to the EGFP transfection group and ${ }^{\#} \mathrm{P}<0.05$, relative to the B19V-VP1u transfection group.

those cells transfected with pEGFP. No significant difference in the expression of NF- $\mathrm{KB}$ protein was detected between H9c2 cells that were transfected with pEGFP-HBoV-VP1u, pEGFP-B19-VP1uD175A or pEGFP-HBoV-VP1uV12A, and those cells transfected with pEGFP. The lower panel in Fig. 5 shows quantitative results concerning NF- $\mathrm{kB}$ levels normalized to $\beta$-actin.

\section{Discussion}

B19 and $\mathrm{HBoV}$ are the only established pathogenic parvoviruses in the literature, and are responsible for many diseases in human beings and thought to circulate globally. Although both B19-VP1u and HBoV-VP1u exhibit sPLA2-like activity (27), only B19-VP1u has been associated with cardiac diseases $(22,23)$. The present investigation, to the best of our knowledge, is the first to evaluate the involvement of HBoV-VP1u in inducing cardiac inflammatory cytokines, and the difference between B19-VP1u and HBoV-VPlu with respect to induced cytokine profiles. B19-VPlu was found herein to induce significant expression of inflammatory chemokines and cytokines, including MCP2, IP-10, IL-1 $\beta$, IL- 6 and TNF- $\alpha$, in H9c2 cells, whereas HBoV-VP1u only induced significant expression of IL-1 $\beta$ and IL- 6 in the H9c2 cells. These findings demonstrate for the first time the effect of HBoV-VPlu on cardiac inflammation and the different 
A
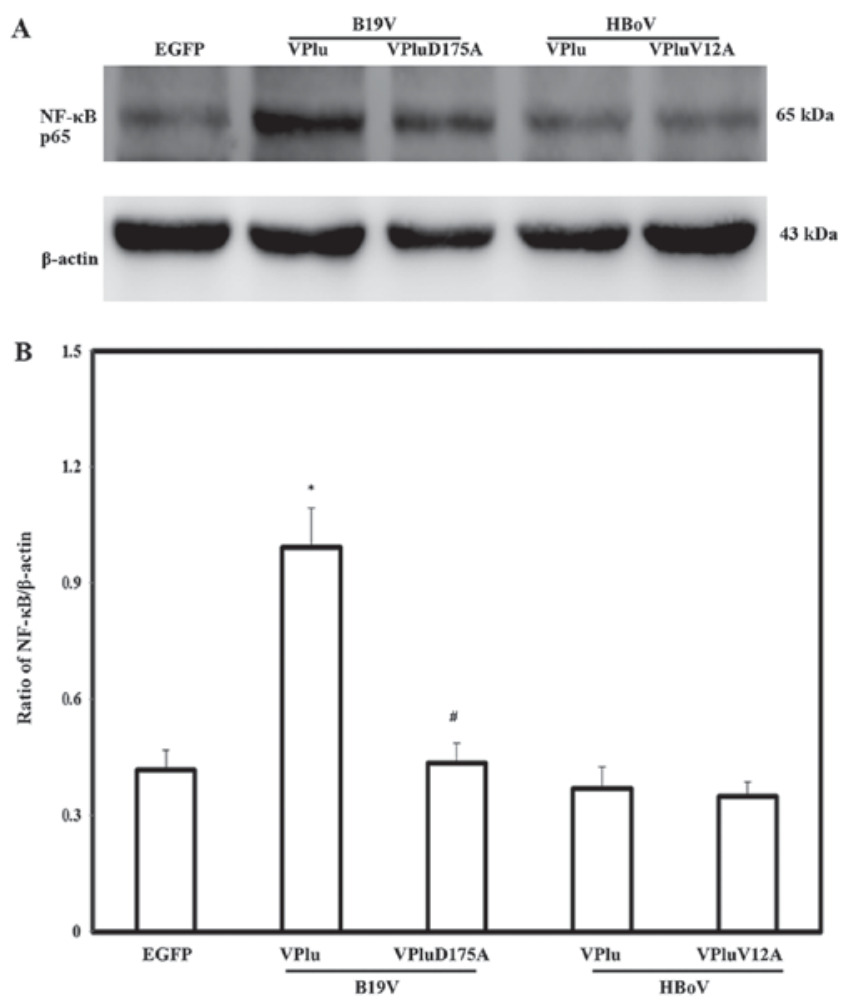

Figure 5. Expression of NF- $\mathrm{KB}$ protein. (A) NF- $\mathrm{KB}$ expression in $\mathrm{H} 9 \mathrm{c} 2$ cells transfected with pEGFP, pEGFP-B19-VP1u, pEGFP-B19-VP1uD175A, pEGFP-HBoV-VP1u or pEGFP-HBoV-VP1uV12A, detected by immunoblotting. (B) The relative amount of NF- $\mathrm{kB}$ is shown based on $\beta$-actin expression. Similar results were observed in triplicate experiments. " $\mathrm{P}<0.05$, relative to the EGFP transfection group and ${ }^{~} \mathrm{P}<0.05$, relative to the $\mathrm{B} 19 \mathrm{~V}-\mathrm{VP} 1 \mathrm{u}$ transfection group.

cytokine profiles induced by B19-VP1u and HBoV-VP1u in H9c2 cells.

The acute response to heart injury involves the production of inflammatory cytokines; however, sustained and long-term inflammation is a primary cause of further damage that can manifest as cardiac hypertrophy and chronic heart failure (30). Both experimental and clinical studies have shown that increased levels of inflammatory cytokines, including tumor necrosis factor (TNF)- $\alpha$, interleukin (IL)-1 $\beta$ and IL-6, are important in the pathogenesis of chronic heart injuries, contributing to cardiac remodeling by influencing hypertrophy, fibrosis and apoptosis (31). Although all of these cytokines are involved in cardiac disorders, various results indicate that the healthy heart does not express TNF, whereas the failing heart generates substantial levels (32), suggesting a role of TNF- $\alpha$ in more severe cardiac disorder. Therefore, various chemokines, such as CXCL10 (IP-10) and CCL8 (MCP2), may also be associated with various heart disorders, including atherosclerosis, ischemia of the myocardium and cardiac fibrosis (33-35). Over recent decades, mounting evidence has indicated a pathogenic role of B19 in various cardiac disorders, including myocarditis, acute myocardial infarction, left ventricular dysfunction, inflammatory cardiomyopathy and heart failure (10,12-16). In this study, B19-VP1u induced significant expression of MCP2, IP-10, TNF- $\alpha$, IL-1 $\beta$ and IL- 6 in H9c2 cells and HBoV-VP1u induced significant expression of IL-1 $\beta$ and IL-6. Therefore, although B19-VP1u and HBoV-VP1u may differ in their induction of proinflammatory factors in $\mathrm{H} 9 \mathrm{c} 2$ cells, the above results imply that both B19-VP1u and HBoV-VP1u are involved in cardiac disorders. However, further investigations must be performed to verify the precise role of HBoV-VPlu in cardiac injuries.

B19 infection has been strongly associated with sPLA2 activity, mediated by its VPlu region. Meanwhile, a previous study reported that VP1u in HBoV exhibited sPLA2-like enzymatic activity (25). The sPLA2-like motif of the VP1-unique (VP1u) region of B19 and $\mathrm{HBoV}$ has been demonstrated to be critical to B19 neutralization and infectivity $(2,17-19,27,36)$. However, the role of sPLA2-like activity in the potential pathogenic function of $\mathrm{HBoV}$ in cardiac injury remains unclear. In this study, different profiles of inflammatory indicators were induced in H9c2 cells by B19-VP1u and HBoV-VP1u, respectively. Notably, no induction of the inflammatory indicators was observed in $\mathrm{H} 9 \mathrm{c} 2$ cells that were transfected with the mutant forms of B19-VP1u and HBoV-VP1u. These findings demonstrate, to the best of our knowledge for the first time, the critical role of the sPLA2 activity of B19-VP1u and HBoV-VPlu in inducing cardiac inflammatory cytokines.

Interestingly, the chemokine MCP-2 and IP-10 were not significantly induced in $\mathrm{H} 9 \mathrm{c} 2$ cells that were transfected with pEGFP-HBoV-VPlu, indicating the possibility that the VP1-u region of $\mathrm{HBoV}$ has a different specificity and only mildly affects the induction of chemokines in cardiac cells. However, further work is required to clarify the precise mechanism of HBo-VP1u in cardiac cells. Overall, B19-VP1u probably has a more prominent role than HBoV-VPlu in the inflammatory responses that are associated with cardiac injury.

\section{Acknowledgements}

Not applicable.

\section{Funding}

The present study was funded by the Chung Shan Medical University and Chi-Mei Medical Center cooperative project (grant no. CMCSMU10502). Consumptive materials were partially supported by the National Science Council (grant nos. NSC99-2320-B-040-007-MY3 and NSC101-2314-B-040-008). The funders had no role in study design, data collection and analysis, decision to publish, or preparation of the manuscript.

\section{Availability of data and materials}

All data generated or analyzed during this study are included in this published article.

\section{Authors' contributions}

HPH was involved in the study conception and design, drafting and revising of the manuscript, and analysis of data. CCC was involved in drafting of the manuscript, study conception and design, and performing experiments. DWL and YFS performed experiments. TCH and BST was involved in the study conception and design, drafting and revising of the manuscript, analysis of data and study supervision. 


\section{Ethics approval and consent to participate}

Not applicable.

\section{Patient consent for publication}

Not applicable.

\section{Competing interests}

The authors declare that they have no competing interests.

\section{References}

1. Berns KI and Parrish CR: Parvoviridae. In: Fields Virology. Knipe DM, Howley PM, Cohen JI, Griffin DE, Lamb RA Martin MA, Racaniello VR and Roizman B (eds). 6th edition. Lippincott Williams \& Wilkins, Philadelphia, PA, pp1768-1791, 2013.

2. Qiu J, Söderlund-Venermo M and Young NS: Human parvoviruses. Clin Microbiol Rev 30: 43-113, 2017.

3. Cossart YE, Field AM, Cant B and Widdows D: Parvovirus-like particles in human sera. Lancet 1: 72-73, 1975.

4. Brown KE and Young NS: Parvovirus B19 in human disease. Annu Rev Med 48: 59-67, 1997.

5. Heegaard ED and Brown KE: Human parvovirus B19. Clin Microbiol Rev 15: 485-505, 2002.

6. Young NS and Brown KE: Parvovirus B19. N Engl J Med 350: 586-597, 2004

7. Page C, François C, Goëb V and Duverlie G: Human parvovirus B19 and autoimmune diseases. Review of the literature and pathophysiological hypotheses. J Clin Virol 72: 69-74, 2015.

8. Allander T, Tammi MT, Eriksson M, Bjerkner A, Tiveljung-Lindell A and Andersson B: Cloning of a human parvovirus by molecular screening of respiratory tract samples. Proc Natl Acad Sci USA 102: 12891-12896, 2005.

9. Jartti T, Hedman K, Jartti L, Ruuskanen O, Allander T and Söderlund-Venermo M: Human bocavirus-the first 5 years. Rev Med Virol 22: 46-64, 2012.

10. Naides SJ and Weiner CP: Antenatal diagnosis and palliative treatment of non-immune hydrops fetalis secondary to fetal parvovirus B19 infection. Prenat Diagn 9: 105-114, 1989.

11. Feldman AM and McNamara D: Myocarditis. N Engl J Med 343 : 1388-1398, 2000.

12. Murry CE, Jerome KR and Reichenbach DD: Fatal parvovirus myocarditis in a 5-year-old girl. Hum Pathol 32: 342-345, 2001.

13. Lamparter S, Schoppet M, Pankuweit S and Maisch B: Acute parvovirus B19 infection associated with myocarditis in an immunocompetent adult. Hum Pathol 34: 725-728, 2003.

14. Kühl U, Pauschinger M, Bock T, Klingel K, Schwimmbeck CP, Seeberg B, Krautwurm L, Poller W, Schultheiss HP and Kandolf R: Parvovirus B19 infection mimicking acute myocardial infarction. Circulation 108: 945-950, 2003.

15. Pankuweit S, Moll R, Baandrup U, Portig I, Hufnagel G and Maisch B: Prevalence of the parvovirus B19 genome in endomyocardial biopsy specimens. Hum Pathol 34: 497-503, 2003.

16. Pankuweit S, Lamparter S, Schoppet $M$ and Maisch B Parvovirus B19 genome in endomyocardial biopsy specimen. Circulation 109: e179, 2004.

17. Dorsch S, Liebisch G, Kaufmann B, von Landenberg P, Hoffmann JH, Drobnik W and Modrow S: The VP1 unique region of parvovirus $\mathrm{B} 19$ and its constituent phospholipase A2-like activity. J Virol 76: 2014-2018, 2002.

18. Filippone C, Zhi N, Wong S, Lu J, Kajigaya S, Gallinella G, Kakkola L, Söderlund-Venermo M, Young NS and Brown KE: VP1u phospholipase activity is critical for infectivity of full-length parvovirus B19 genomic clones. Virology 374: 444-452, 2008
19. Leisi R, Ruprecht N, Kempf C and Ros C: Parvovirus B19 uptake is a highly selective process controlled by VP1u, a novel determinant of viral tropism. J Virol 87: 13161-13167, 2013.

20. Hsu TC, Tsai CC, Chiu CC, Hsu JD and Tzang BS: Exacerbating effects of human parvovirus B19 NS1 on liver fibrosis in NZB/W F1 mice. PLoS One 8: e68393, 2013.

21. Tsai CC, Chiu CC, Hsu JD, Hsu HS, Tzang BS and Hsu TC Human parvovirus B19 NS1 protein aggravates liver injury in NZB/W F1 mice. PLoS One 8: e59724, 2013

22. Nie X, Zhang G, Xu D, Sun X, Li Z, Li X, Zhang X, He F and Li Y The VP1-unique region of parvovirus B19 induces myocardial injury in mice. Scand J Infect Dis 42: 121-128, 2010.

23. Tzang BS, Lin TM, Tsai CC, Hsu JD, Yang LC and Hsu TC: Increased cardiac injury in NZB/W F1 mice received antibody against human parvovirus B19 VP1 unique region protein. Mol Immunol 48: 1518-1524, 2011.

24. Bogomolovas J, Šimoliūnas E, Rinkūnaitè I, Smalinskaitė L, Podkopajev A, Bironaitè D, Weis CA, Marx A, Bukelskienė V, Gretz N, et al: A novel murine model of parvovirus associated dilated cardiomyopathy induced by immunization with VP1-unique region of parvovirus B19. Biomed Res Int 2016: 1627184,2016

25. Qu XW, Liu WP, Qi ZY, Duan ZJ, Zheng LS, Kuang ZZ, Zhang WJ and Hou YD: Phospholipase A2-like activity of human bocavirus VP1 unique region. Biochem Biophys Res Commun 365: 158-163, 2008

26. Tzang BS, Tsay GJ, Lee YJ, Li C, Sun YS and Hsu TC: The association of VP1 unique region protein in acute parvovirus B19 infection and anti-phospholipid antibody production. Clin Chim Acta 378: 59-65, 2007.

27. Chiu CC, Shi YF, Yang JJ, Hsiao YC, Tzang BS and Hsu TC: Effects of human Parvovirus B19 and Bocavirus VP1 unique region on tight junction of human airway epithelial A549 cells. PLoS One 9: e107970, 2014.

28. Zádori Z, Szelei J, Lacoste MC, Li Y, Gariépy S, Raymond P, Allaire M, Nabi IR and Tijssen P: A viral phospholipase A2 is required for parvovirus infectivity. Dev Cell 1: 291-302, 2001.

29. Bonnerot C, Rocancourt D, Briand P, Grimber G and Nicolas JF: A beta-galactosidase hybrid protein targeted to nuclei as a marker for developmental studies. Proc Natl Acad Sci USA 84: 6795-6799, 1987

30. Valaperti A: Drugs targeting the canonical NF- $\kappa B$ pathway to treat viral and autoimmune myocarditis. Curr Pharm Des 22: 440-449, 2016

31. Gullestad L, Ueland T, Vinge LE, Finsen A, Yndestad A and Aukrust P: Inflammatory cytokines in heart failure: Mediators and markers. Cardiology 122: 23-35, 2012.

32. Feldman AM, Combes A, Wagner D, Kadakomi T, Kubota T, Li YY and McTiernan C: The role of tumor necrosis factor in the pathophysiology of heart failure. J Am Coll Cardiol 35: 537-544, 2000.

33. Frangogiannis NG: Chemokines in the ischemic myocardium: From inflammation to fibrosis. Inflamm Res 53: 585-595, 2004

34. Braunersreuther V, Mach F and Steffens S: The specific role of chemokines in atherosclerosis. Thromb Haemost 97: 714-721, 2007.

35. Ardigo D, Assimes TL, Fortmann SP, Go AS, Hlatky M, Hytopoulos E, Iribarren C, Tsao PS, Tabibiazar R and Quertermous T; ADVANCE Investigators: Circulating chemokines accurately identify individuals with clinically significant atherosclerotic heart disease. Physiol Genomics 31: 402-409, 2007.

36. Ros C, Gerber M and Kempf C: Conformational changes in the VP1-unique region of native human parvovirus B19 lead to exposure of internal sequences that play a role in virus neutralization and infectivity. J Virol 80: 12017-12024, 2006. 